\title{
Type 2 diabetes after gestational diabetes: greater than fourfold risk among Indigenous compared with non-Indigenous Australian women ${ }^{\dagger}$
}

\author{
Q1 Catherine R. Chamberlain $1,2 *$ \\ Brian Oldenburg ${ }^{1}$ \\ Alyce N. Wilson ${ }^{1}$ \\ Sandra Eades ${ }^{3}$ \\ Kerin O'Dea ${ }^{4}$ \\ Jeremy Oats ${ }^{1}$ \\ Rory Wolfe ${ }^{2}$
}

Q2 ${ }^{1}$ Melbourne School of Population and Global Health, The University of Melbourne, Melbourne, VIC, Australia

${ }^{2}$ Department of Epidemiology and Preventive Medicine, Monash University, Melbourne, VIC, Australia

${ }^{3}$ Baker IDI Heart and Diabetes Institute, Melbourne, VIC, Australia

${ }^{4}$ School of Population Health, University of South Australia, Adelaide, SA, Australia

*Correspondence to: Catherine Chamberlain, Melbourne School of Population and Global Health, The University of Melbourne, Rm 421, Level 4, 207 Bouverie Street, Melbourne, VIC 3010, Australia. E-mail: Catherine.chamberlain@ unimelb.edu.au

'The term 'Indigenous' is used when referring to Aboriginal and Torres Strait Islander peoples in Australia collectively and Indigenous peoples in other countries. This is for ease of reading in this paper article only, and we respectfully acknowledge the diversity and autonomy of different communities included in this broad term.

Received: 20 April 2015

Revised: 6 July 2015

Accepted: 28 July 2015

\begin{abstract}
Background Gestational diabetes is associated with a high risk of type 2 diabetes. However, progression rates among Indigenous women in Australia who experience high prevalence of gestational diabetes are unknown.

Methods This retrospective cohort study includes all births to women at a regional hospital in Far North Queensland, Australia, coded as having 'gestational diabetes' from 1 January 2004 to 31 December 2010 (1098 births) and receiving laboratory postpartum screening from 1 January 2004 to 31 December 2011 ( $n=483$ births). Women who did not receive postpartum screening were excluded from the denominator. Data were linked between hospital electronic records, routinely collected birth data and laboratories, with sample validation by reviews of medical records. Analysis was conducted using Coxproportional regression models.
\end{abstract}

Results Indigenous women had a greater than fourfold risk of developing type 2 diabetes within 8 years of having gestational diabetes, compared with non-Indigenous women (hazards ratio 4.55, 95\% confidence interval 2.63$7.88, p<0.0001)$. Among women receiving postpartum screening tests, by 3 , 5 and 7 years postpartum, 21.9\% (15.8-30.0\%), 25.5\% (18.6-34.3\%) and $42.4 \%$ (29.6-58.0\%) Indigenous women were diagnosed with type 2 diabetes after gestational diabetes, respectively, compared with 4.2\% (2.5-7.2\%), 5.7\% (3.3-9.5\%) and $13.5 \%(7.3-24.2 \%)$ non-Indigenous women. Multivariate analysis showed significant associations between an increased rate of type 2 diabetes with an early pregnancy body mass index $>25 \mathrm{~kg} / \mathrm{m}^{2}$, partial breastfeeding only at hospital discharge and gestational diabetes diagnosis prior to 17 weeks gestation.

Conclusions This study demonstrates that, compared with non-Indigenous women, Indigenous Australian women have a greater than fourfold risk of developing type 2 diabetes after gestational diabetes. Strategies are urgently needed to reduce rates of type 2 diabetes by supporting a healthy weight and breastfeeding and to improve postpartum screening among Indigenous women with gestational diabetes. Copyright (C) 2015 John Wiley \& Sons, Ltd.

Keywords gestational diabetes mellitus; type 2 diabetes mellitus; diabetes; pregnancy; aboriginal; Indigenous 
Abbreviations BMI, body mass index; CHCCS, Cairns Hospital Clinical Coding system; FPG, fasting plasma glucose; Gestational diabetes, gestational diabetes mellitus; $\mathrm{HbA}_{1 \mathrm{c}}$, glycosylated haemoglobin; HR, hazards ratio; MPDC, Midwives Perinatal Data Collection; OGTT, oral glucose tolerance test; Type 2 diabetes, type 2 diabetes mellitus

\section{Introduction}

Gestational diabetes mellitus (gestational diabetes), defined as diabetes diagnosed during pregnancy [1], is increasing in prevalence, with particularly high rates reported among Indigenous women worldwide [2]. In addition to causing serious complications in pregnancy and birth [3], women diagnosed with gestational diabetes have a very high risk of developing type 2 diabetes mellitus (type 2 diabetes) postpartum [4]. If left undetected and untreated in the longer term, unmanaged hyperglycaemia associated with type 2 diabetes can cause serious complications in subsequent pregnancies [5] and lead to multiple comorbidities for the mother [6].

Particularly high rates of progression from gestational to type 2 diabetes have been reported among Indigenous women internationally [7]. Progression rates range from $30 \%$ to $70 \%$ within 4 years postpartum, among Indigenous women in Canada [8], New Zealand [9] and the United States $[10,11]$. However, there are currently few studies investigating rates of progression from gestational to type 2 diabetes among Aboriginal and Torres Strait Islander (Indigenous) women in Australia. One study reported that two out of seven Indigenous Australian women with gestational diabetes receiving postpartum screening had developed type 2 diabetes, but this sample was too small for substantive analysis [12].

The aim of this article is to investigate the rates and associations with progression to type 2 diabetes among Indigenous and non-Indigenous women, diagnosed with gestational diabetes in Far North Queensland (Australia). Comparisons between Indigenous and non-Indigenous women are also needed to determine whether existing services currently provided for all women are adequate for Indigenous women or whether tailoring of services and additional strategies are required for Indigenous women.

\section{Materials and methods}

\section{Study setting and sample}

This study includes all women who gave birth at Cairns Hospital and were coded as having gestational diabetes from 1 January 2004 to 31 December 2010. Cairns Hospital is the only secondary referral hospital for Far North Queensland, a vast region on the northeast tip of Australia covering almost $300000 \mathrm{~km}^{2}$. The region has a population of over 230 000, and approximately 40000 (17\%) are Aboriginal and Torres Strait Islander people [13]. About half of the population lives in the main regional centre of Cairns, and the remainder lives in areas classified as rural and remote, in a sparsely populated tropical region that has limited sealed road access and is subject to extreme weather events. More than $80 \%$ of women in the region give birth at Cairns Hospital, which includes almost all women with gestational diabetes. The study setting and design details are reported in detail elsewhere [14].

The study is a retrospective cohort design that used linked electronic data validated by medical record reviews. The following data sources were linked: (1) the Cairns Hospital Clinical Coding system (CHCCS) entries for all women who gave birth at Cairns Hospital between 1 January 2004 and 31 December 2010 and were coded as having gestational diabetes (International Classification Diseases codes 024.41, 024.42, 024.42, 0.24 .43 and 0.24.44); (2) pregnancy and birth details from the Midwives Perinatal Data Collection (MPDC); and (3) postpartum glucose test details from the three local laboratories. Additionally, available medical records were reviewed for all births among Indigenous women $(n=578)$ and a random sample of births among non-Indigenous women $(n=332)$ to validate gestational diabetes coding and extract additional data not reliably available in electronic data [antepartum and postpartum care providers, body mass index (BMI) and English proficiency]. This sample size was assessed as adequate to detect a $10 \%$ difference in postpartum screening between Indigenous and nonIndigenous women.

\section{Gestational diabetes diagnostic criteria}

During the study period, the diagnostic criteria for gestational diabetes were consistent with the Australian Diabetes in Pregnancy Society guidelines [15], which required fasting plasma glucose (FPG) $\geq 5.5 \mathrm{mmol} / \mathrm{L}$ or 2-h glucose $\geq 8 \mathrm{mmol} / \mathrm{L}$ following a 75 -g oral glucose tolerance test (OGTT). However, the standard procedure for gestational diabetes screening varied markedly across Australia during this period [16]. In 2005, local primary care guidelines were developed, which recommended a random blood glucose level at each pregnancy visit, and if the level was $\geq 5.0 \mathrm{mmol} / \mathrm{L}$, a fasting blood glucose level was offered [17]. At 24 weeks, a random blood glucose level was offered again, and if $\geq 5.0 \mathrm{mmol} / \mathrm{L}$ or the woman was classified as 'at risk' of gestational diabetes, a 75-g OGTT was offered. 'At risk' included a history of 
unexplained miscarriage or stillbirth, large baby $(>4000 \mathrm{~g}$ or 'large for dates'), age $>30$ years, obesity (BMI $>30 \mathrm{~kg} / \mathrm{m}^{2}$ ), previous neonatal hyperglycaemia, family history of diabetes, past history of gestational diabetes or polycystic ovary syndrome (indigeneity was not a risk factor). If normal, the 75-g OGTT was to be offered again at 32 weeks. Glycosylated haemoglobin $\left(\mathrm{HbA}_{1 \mathrm{c}}\right)$ was recommended around 28 weeks or at first presentation. In 2007, the primary care guidelines changed to include all Indigenous women as 'high risk', along with 'other high risk populations', such as Pacific Islander, Chinese and Mediterranean people, and recommended a 75g OGTT at 26-30 weeks gestation [18]. In 2009, the guidelines were further revised to recommend a 75-g OGTT at 24-28 weeks gestation [19], as per the Australian Diabetes in Pregnancy Society guidelines [15].

\section{Type 2 diabetes diagnostic criteria}

The diagnostic criteria for type 2 diabetes at the time of T1 the study are outlined in Table 1, which was clarified in consultation with local endocrinology experts. The recommended diagnosis was based on glucose readings following a 75-g OGTT equal to or more than $7 \mathrm{mmol}$ (fasting), $10 \mathrm{mmol} / \mathrm{L}$ (after $1 \mathrm{~h}$ ) or $11 \mathrm{mmol} / \mathrm{L}$ (after $2 \mathrm{~h}$ ) [20]. In this study, women with a diagnosis of type 2 diabetes were identified by the presence of one of these readings, a notation on the laboratory results identifying the woman as having type 2 diabetes, subsequent coding of a pregnancy as type 2 diabetes or documentation of type 2 diabetes in the pregnancy or medical records.

\section{Indigenous status criteria}

Indigenous status is a measure of whether a person selfidentifies as being of Aboriginal, Torres Strait Islander or Aboriginal and Torres Strait Islander descent, and this is recorded in the CHCCS.

\section{Data analysis}

De-identified data were exported from Microsoft Access for analysis in Stata 13 [21]. All analyses were stratified by Indigenous status. Primary analyses were restricted to women who received a laboratory-based postpartum screening test (OGTT, $\mathrm{HbA}_{1 \mathrm{c}}, \mathrm{FPG}$ or random plasma glucose) as women who did not receive a test had no chance of being diagnosed with type 2 diabetes. Analyses included all births to mothers coded as having gestational diabetes, who received a laboratory-based postpartum screening test, where routinely collected data on potential moderating factors were consistently available in electronic data, including country of birth, remoteness, maternal age, number of antenatal care visits, parity, smoking during pregnancy, medical complications, induction of labour, mode of birth and confinement date (Table 1). Analyses were restricted only to those records where the medical records were reviewed when data were not consistently recorded in routine electronic data, including BMI at first antenatal visit, location of antenatal or postnatal care (hospital, private general practitioner clinic, government clinic, community-controlled health service or other), breastfeeding at discharge and diagnosis of gestational diabetes prior to 17 weeks gestation ('probable type 2 diabetes') (Table 2). Sensitivity analyses were also T2 conducted including all eligible women with gestational diabetes, with and without postpartum screening, in the denominator.

The time to type 2 diabetes diagnosis from confinement date, among births to mothers coded as having gestational diabetes, was summarized using Kaplan-Meier survival estimates and analysed using Cox-proportional hazards regression models. Women were censored from the analysis if they became pregnant and once type 2 diabetes was diagnosed. The censorship was calculated from time of onset of subsequent pregnancy, calculated as 273 days prior to subsequent confinement or 20 weeks prior to date of test if the test was coded as 'during pregnancy' and no pregnancy was recorded (including

Table 1. Glucose parameters used during study period in Far North Queensland ${ }^{\mathrm{a}}$ [20]

\begin{tabular}{|c|c|c|c|c|c|c|c|}
\hline \multirow{2}{*}{ Test } & \multirow{2}{*}{$\begin{array}{l}\text { Glucose } \\
\text { load (g) }\end{array}$} & \multicolumn{2}{|c|}{$\begin{array}{l}\text { Normal reference } \\
\text { range }(\mathrm{mmol} / \mathrm{L})\end{array}$} & \multicolumn{2}{|c|}{$\begin{array}{l}\text { Impaired glucose } \\
\text { tolerance (mmol/L) }\end{array}$} & \multicolumn{2}{|c|}{$\begin{array}{l}\text { Abnormal glucose } \\
\text { tolerance (mmol/L) }\end{array}$} \\
\hline & & Pregnant & $\begin{array}{c}\text { Not } \\
\text { pregnant }\end{array}$ & Pregnant & Not pregnant & Pregnant & $\begin{array}{l}\text { Not } \\
\text { pregnant }\end{array}$ \\
\hline OGTT fasting/FPG & 75 & $3.6-5.4$ & $3.6-5.4$ & $5.5-6.9$ & $5.5-6.9$ & $\geq 7.0$ & $\geq 7.0$ \\
\hline OGTT $2 \mathrm{~h}$ & 75 & $<5.5$ & $<7.8$ & $5.5-7.9$ & $\begin{array}{c}7.8-11.0 \\
(a b n o r m a l \\
\text { if FPG also >6.0) }\end{array}$ & $\geq 8.0$ & $\geq 11.0$ \\
\hline
\end{tabular}

FPG, fasting plasma glucose; OGTT, oral glucose tolerance test.

${ }^{a}$ Modified in consultation with local endocrinology experts about criteria used during study period. 


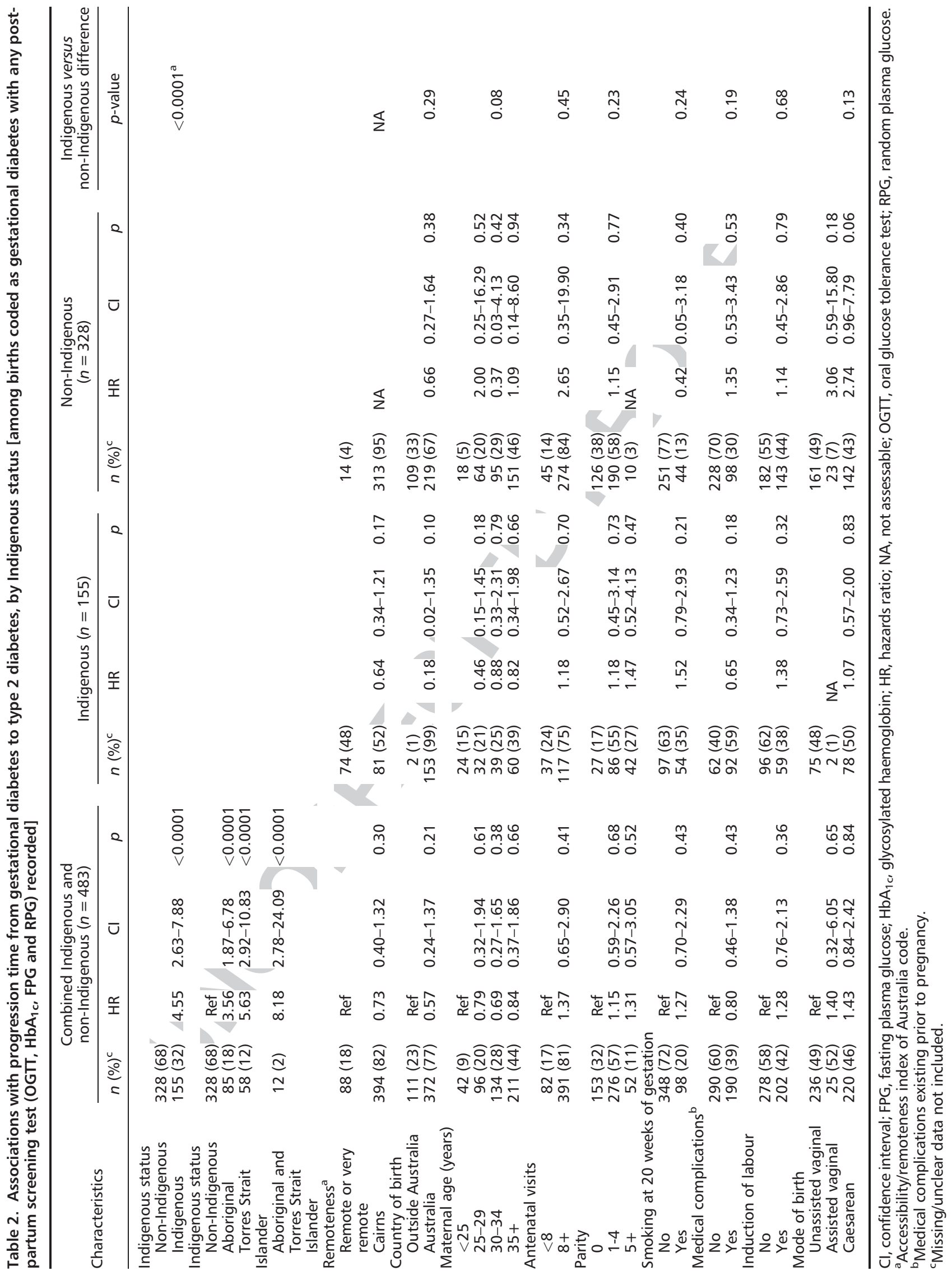


all tests after 1 March 2010). The date of type 2 diabetes diagnosis was calculated from the data of any records identifying type 2 diabetes from any data source (e.g. medical records, MPDC data and laboratory tests). The Cox-proportional hazards regression models were extended using interaction terms to investigate possible effect modification of time to type 2 diabetes diagnosis between Indigenous and non-Indigenous women, with likelihood ratio tests used to calculate a single $p$-value where there are multiple categories of continuous variables. Throughout, two-tailed tests were conducted, and $p<0.05$ was considered statistically significant.

\section{Ethics}

This project was conducted with assistance from Cairns Diabetes Centre and Apunipima Cape York Health Council. Governance was provided by a project advisory group that included Indigenous researchers and representatives of Apunipima Cape York Health Council (community-controlled organization). Ethical approval was granted for this project by the Cairns Hospital and Hinterland Research Ethics Committee, the Monash University Human Research and Ethics Committee, and the Queensland Health Research Ethics and Governance Unit (no. 201101190).

\section{Results}

From 1 January 2004 to 31 December 2010, 1012 women were identified in the CHCCS as giving birth at Cairns Hospital and coded as having an episode of gestational diabetes, from a total of 16765 births during the same period. This includes 352 Indigenous women and 660 non-Indigenous women. These women had 1505 births during the study period, and 1098 of these births were coded as gestational diabetes after linkage with MPDC data and medical record review of 912 pregnancies (crude gestational diabetes prevalence $=6.5 \%$ ). Approximately $6 \%$ of births where medical records were reviewed were reclassified as gestational diabetes, and hence, gestational diabetes case ascertainment is likely to be underestimated by approximately 37 cases in this sample, detailed elsewhere [22]. Two women who died in the early postpartum period were excluded from analysis. Postpartum screening tests were available following 483/1098 births coded as gestational diabetes, and analysis of type 2 diabetes progression was restricted to this subset with F1 postpartum tests recorded (Figure 1). Compared with women who did not receive postpartum screening, Indigenous women who received postpartum screening were more likely to live in remote areas, and non-Indigenous women who received screening were more likely to be born outside Australia, be older, have more than five previous pregnancies, not smoke or breastfeed [23]. There were no type 2 diabetes diagnoses recorded among births where no postpartum screening test was available. There were 82 type 2 diabetes diagnoses reported following births coded as having gestational diabetes: 76 identified by laboratory test results and six identified in clinical coding or medical records, where the subsequent pregnancy was coded as type 2 diabetes. As previously reported [22], Indigenous women had significantly longer times to postpartum screening compared with non-Indigenous women.

\section{Rates of progression from gestational diabetes to type 2 diabetes}

Among women who received a postpartum screening test, Indigenous women with gestational diabetes had a greater than fourfold risk of developing type 2 diabetes [hazards ratio (HR) 4.55, 95\% confidence interval $2.63-7.88, p<0.0001]$.

By 3 years postpartum, among the 498/1098 gestational diabetes births where postpartum screening tests were recorded, $21.9 \%(15.8-30.0 \%)$ Indigenous women and $4.2 \%(2.5-7.2 \%)$ non-Indigenous women were diagnosed with type 2 diabetes following gestational diabetes. By 5 years postpartum, 25.5\% (18.6-34.3\%) Indigenous and $5.7 \%(3.3-9.5 \%)$ non-Indigenous women were diagnosed with type 2 diabetes following gestational diabetes. And by 7 years postpartum, 42.4\% (29.6-58.0\%) Indigenous women and $13.5 \%$ (7.3-24.2\%) non-Indigenous women were diagnosed with type 2 diabetes following gestational diabetes (Figure 2).

\section{Sensitivity analysis}

Sensitivity analyses including all eligible women with and without postpartum screening in the denominator continued to show almost a fourfold risk of developing type 2 diabetes among Indigenous women, compared with non-Indigenous women (HR 3.86, 95\% confidence interval 2.24-6.67, $p<0.0001$ ). However, as would be expected given that less than $50 \%$ women received a postpartum screening test, if all women are included in the denominator, the proportions of women progressing to type 2 diabetes were less than half that reported when only women receiving screening were included. By 3 years postpartum, 9.6\% (6.8-13.4\%) Indigenous women and $2.0 \%(1.2-3.4 \%)$ non-Indigenous women were diagnosed with type 2 diabetes. By 5 years postpartum, $11.2 \%$ (8.0$15.5 \%)$ Indigenous women and $2.7 \%(1.6-4.5 \%)$ nonIndigenous women were diagnosed with type 2 diabetes. 
Flow chart of included study participants

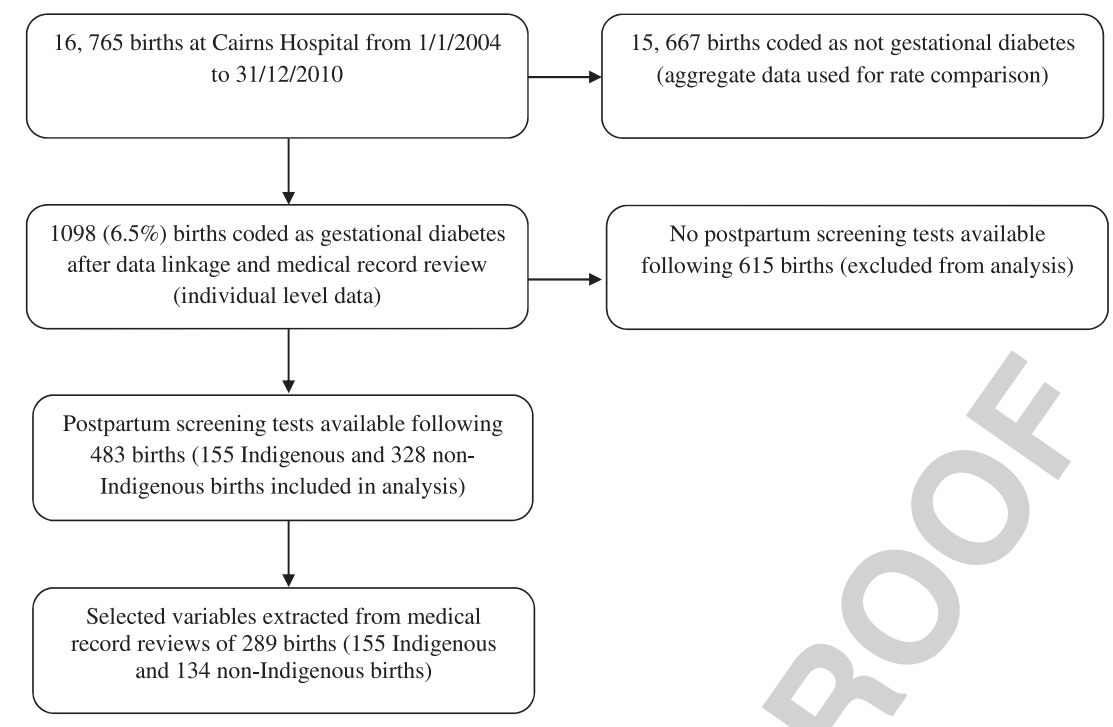

Figure 1. Flow chart of included study participants

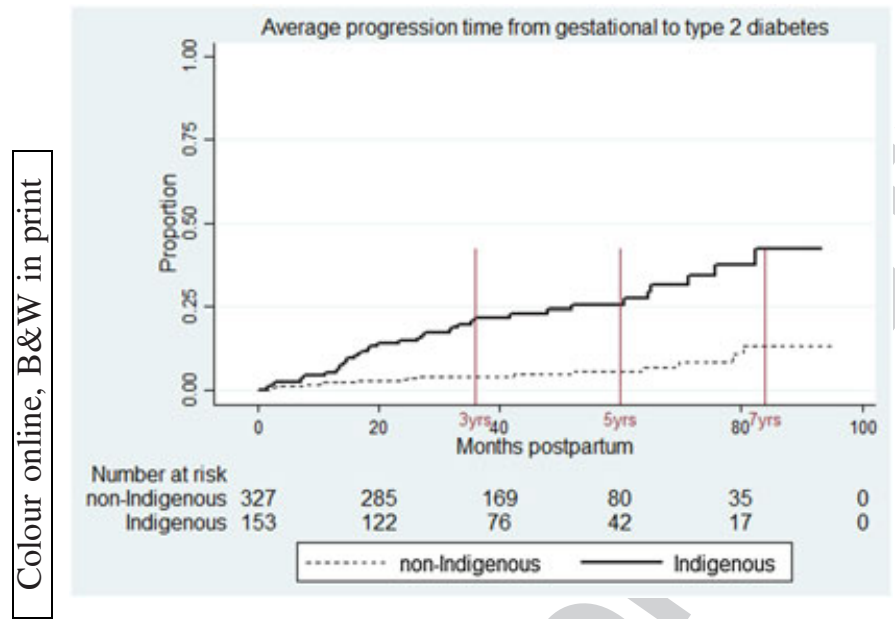

Figure 2. Incidence of type 2 diabetes among women diagnosed with gestational diabetes 1 January 2004 to 31 December 2010, receiving any screening test between 1 January 2004 and 30 November 2011 (Kaplan-Meier estimates)

And by 7 years postpartum, $18.3 \%$ (12.6-26.3\%) Indigenous women and $6.4 \%$ (3.4-11.7\%) non-Indigenous women were diagnosed with type 2 diabetes postpartum (Supporting Information Figure 1).

\section{Factors associated with rates of progression from gestational diabetes to type 2 diabetes}

Indigenous status remained strongly associated with a faster time to progression from gestational diabetes to type 2 diabetes in all multivariate regression analyses $(p<0.0001)$.

In combined analysis, there appeared to be an increased rate of progression from gestational to type 2 diabetes among women with a BMI $>25 \mathrm{~kg} / \mathrm{m}^{2}$ and women partially breastfeeding at discharge from hospital (Table 3). As would be expected, there was also a T3 significantly increased 'risk of progression' among women who were first diagnosed with gestational diabetes prior to 17 weeks gestation and were likely to have undiagnosed type 2 diabetes in pregnancy (Table 3).

Among Indigenous women, there was an increased rate of type 2 diabetes progression among women partially breastfeeding, compared with women fully breastfeeding, at discharge from hospital and among women diagnosed with gestational diabetes prior to 17 weeks gestation ('probable type 2 diabetes'). Despite higher rates in comparison with non-Indigenous women, there were no significant differences in type 2 diabetes progression rates between women who were coded as Aboriginal (HR 3.56, 1.89-6.78), Torres Strait Islander (HR 5.63, 2.92-10.83) or Aboriginal and Torres Strait Islander (HR 8.18, 2.78-24.08) (Table 2; Supporting Information Figure 2).

Among non-Indigenous women, significantly higher rates of type 2 diabetes progression were seen among women who were partially breastfeeding at discharge, compared with those who were fully breastfeeding at discharge from hospital, and women diagnosed with gestational diabetes prior to 17 weeks gestation ('probable type 2 diabetes') (Tables 2 and 3). 


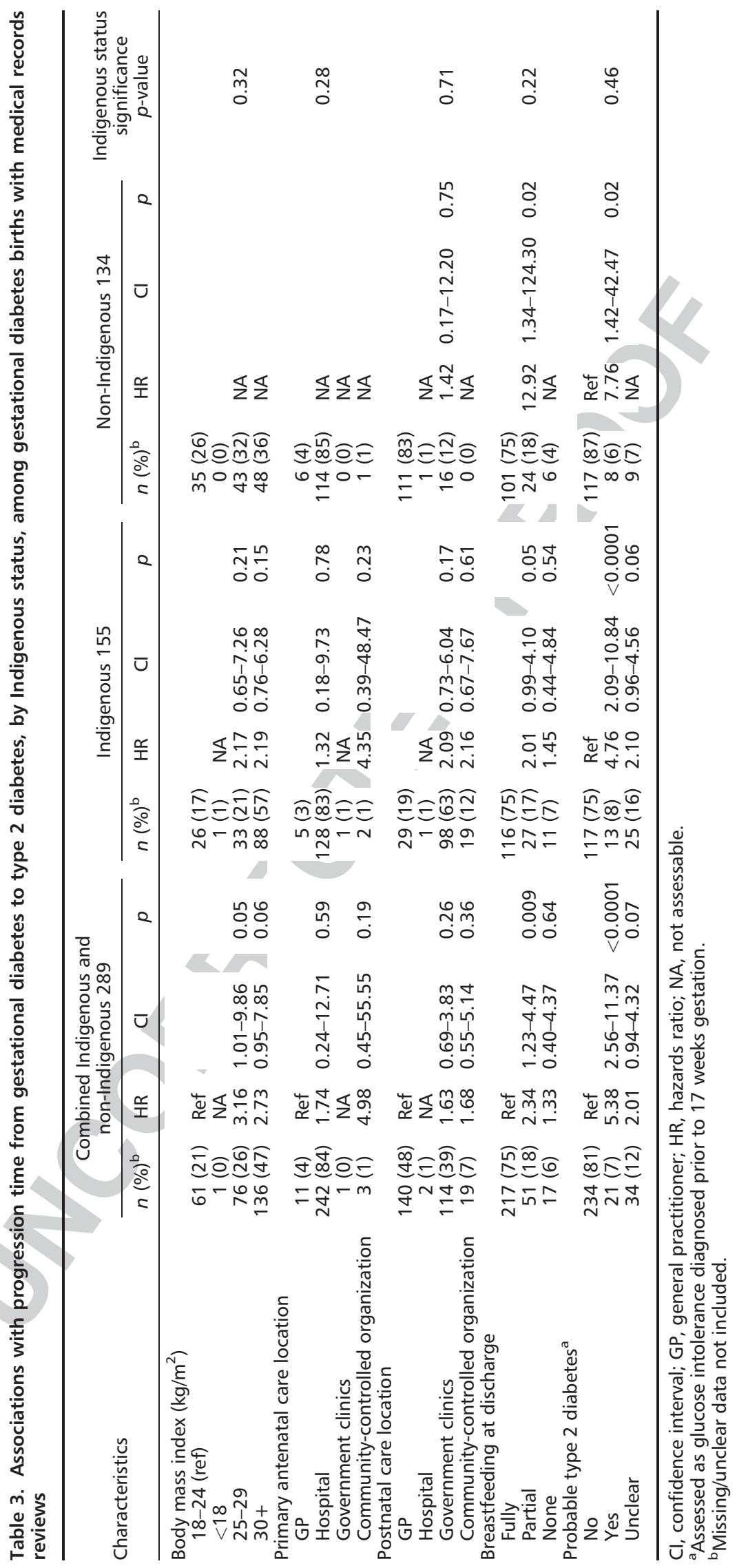




\section{Discussion}

Among women with gestational diabetes who received a laboratory-based postpartum screening test, we found a greater than fourfold risk of progression to type 2 diabetes among Indigenous women compared with non-Indigenous women. Within 5 years postpartum, over 25\% of Indigenous women with gestational diabetes who received a laboratory-based postpartum diabetes screening test had been diagnosed with type 2 diabetes, compared with almost $6 \%$ of non-Indigenous women. There were significantly higher rates of type 2 diabetes progression among women who had a pregnancy BMI $>25$ compared with those with a BMI $<25$, those who were 'partially breastfeeding', compared with those who were 'fully breastfeeding' at discharge from hospital, and women diagnosed with gestational diabetes prior to 17 weeks gestation ('probable type 2 diabetes').

These findings of increased rates of type 2 diabetes progression among Indigenous Australian women are consistent with previous reports of higher rates of type 2 diabetes among Indigenous women internationally [7-11]. Associations with increased risk of type 2 diabetes and both increased pregnancy BMI and not fully breastfeeding at discharge from hospital are also similar to findings reported among non-Indigenous women [4,24].

Major strengths of this study include the use of linked data validated by medical record review to increase the accuracy of gestational diabetes diagnosis coding and restriction of the sample to women who received postpartum diabetes screening tests. However, there are several limitations. First, we were unable to include 'point of care' tests provided by primary healthcare professionals, such as $\mathrm{HbA}_{1}$, FPG and random plasma glucose, using a capillary sample. We identified six type 2 diabetes diagnoses in coding of subsequent pregnancies and medical records, in addition to the 76 identified by laboratory test records. This suggests that it is possible that some additional cases of type 2 diabetes diagnoses may be been missed (diagnosed in other locations or by 'point-of-care-tests'), particularly where there was not a subsequent pregnancy, which would increase the observed rate of progression to type 2 diabetes. However, standard practice in this region is that if glucose intolerance is suspected, a venous sample would be sent to one of the three laboratories included in this study, and all women diagnosed with type 2 diabetes in this study had received postpartum diabetes screening. Second, under-enumeration of Indigenous status recording, with data linkage studies suggesting Indigenous mothers in the NSW MPDC, may be underenumerated by around 40\% [25]. Third, we included women who were diagnosed with gestational diabetes prior to 17 weeks gestation, and these women were highly likely to have had undiagnosed type 2 diabetes prior to pregnancy, reflected in the significantly higher risk of 'progression', and may be increasing the reported risk of progression in this study. Finally, we do not know the rates of progression among women who did not receive a postpartum screening test, and it is difficult to estimate the direction of the effect of this 'selection bias' and whether type 2 diabetes progression rates would be higher or lower among women who did not receive screening. For instance, women in this study who partially breastfed their infants around the time of hospital discharge had higher rates of type 2 diabetes progression than women who fully breastfed, and we had previously reported that 'fully breastfeeding' was associated with a higher likelihood of postpartum screening among non-Indigenous women [23]; hence, inclusion of more women partially breastfeeding could result in a higher observed rate of type 2 diabetes progression. On the other hand, one could argue that clinicians may be likely to more strongly encourage screening among women whom they consider 'at high risk' and that those women who were not screened may have been more likely to be considered 'at low risk'; hence, inclusion of more 'low risk' women would have decreased the observed rate of type 2 diabetes progression. This highlights the need for prospective research that follows up all women with gestational diabetes.

If the rates of progression to type 2 diabetes among women who received postpartum screening are extrapolated to all women in this study who did not receive postpartum screening, by 7 years postpartum, 20 Indigenous women and 13 non-Indigenous women who did not receive postpartum diabetes screening would have undiagnosed and untreated type 2 diabetes. In this study, we contacted the medical service providers for all women who were identified as being due for a postpartum screening test and advised them to offer their clients a postpartum screening test for diabetes if their records did not already indicate that they had received one. These findings highlight the importance of postpartum screening for type 2 diabetes after gestational diabetes, particularly among Indigenous women. This is of particular concern as we have previously reported very low rates of postpartum screening among all women with gestational diabetes in Far North Queensland, with significantly longer time to and lower rates of early postpartum screening among Indigenous women compared with non-Indigenous women [22] and with lower rates among Indigenous women in Cairns compared with women living in remote areas [23]. While this poses a serious risk for women in the longer term, the risks to women of childbearing age include serious risks to subsequent pregnancies, including congenital anomalies.

We have discussed the barriers to postpartum screening previously [23], including issues for women such as 
forgetting, not knowing about the need for a test, inconvenient unpleasant tests, fear of results, time pressures, physician awareness and communication, costs and lack of consistency around postpartum screening guidelines after gestational diabetes for Indigenous women [26]. Recent studies show that some systemic strategies can improve postpartum glucose screening rates among nonIndigenous women [27]. These include case management [28], patient and physician reminders, system changes, proactive postpartum care plans, antenatal education, registers, clinical protocols and electronic records [27]. The effectiveness of these strategies has led to calls for them to be a part of routine postpartum care for women with gestational diabetes [29]. However, given the low rates of postpartum screening among Indigenous women [22], there is a need to assess the specific needs of women and assess whether cultural tailoring or additional strategies are required.

While there has been considerable debate about gestational diabetes care and treatment pathways during pregnancy, guidelines for postpartum care for women at high risk of developing type 2 diabetes have lagged behind [30], and opportunities for prevention are being lost. A diagnosis of gestational diabetes offers a unique 'window of opportunity' to identify women at increased risk of developing type 2 diabetes and offer effective support. The increased insulin resistance occurring as a result of naturally occurring pregnancy hormones offers a "natural stress test' to identify women with impaired glucose tolerance, who may have not been identified had they not become pregnant. Further, frequent scheduled contacts with health services during and after pregnancy provide an additional opportunity to support women to reduce the risk of developing type 2 diabetes for themselves and their infant, by breastfeeding and healthy lifestyle changes, with any effective support having significant benefits for many generations to follow [31]. Finally, studies suggest that women with gestational diabetes are likely to be highly motivated during the postpartum period to improve the health of their infant and family, with a heightened state of 'change readiness' [32]. However, while pregnancy and the postpartum period afford opportunities to reduce the risk of type 2 diabetes, there are also a number of challenges including tiredness, maternal attachment and childcare demands in the early postpartum period, work, family and child development in subsequent years [33] and postpartum depression [34]. Several recent studies also suggest that pregnancy care providers lack confidence in talking to women about obesity-related issues [35].

Hence, well-designed strategies to address the needs of women and ensure provision of professional support are essential [36]. Results in this study reinforce calls for supporting a healthy BMI and breastfeeding as important prevention strategies [37]. A recent meta-synthesis of qualitative studies of 'perceptions of women with gestational diabetes' suggested that important factors to overcome these challenges included addressing emotional issues, providing clear advice and offering an intervention that works for the whole family [38]. And while technological interventions have been trialled as convenient options [39], it is likely that personal support will be needed to address emotional issues $[40,41]$. This is likely to be particularly important among Indigenous women with gestational diabetes, as a study in the United States found that Indigenous women with gestational diabetes reported high perceptions of risk coupled with a low sense of self-efficacy [42], a combination associated with avoidance behaviour [43]. This raises questions as to whether traditional risk advice is likely to be helpful and suggests that a strength-based focus that improves self-efficacy may be more effective. This reinforces recommendations in the National Aboriginal and Torres Strait Islander Health Plan [44], which was developed to guide evidence-based strategies to 'close the gap' in health inequities, for strength-based familycentred interventions using life-course approaches that focus on social determinants.

Despite the risks of developing type 2 diabetes among women with previous gestational diabetes, there are few studies demonstrating effective strategies to reduce the risks for women after gestational diabetes $[30,45]$. Importantly, there are no studies investigating strategies to improve postpartum prevention or screening among Indigenous women with gestational diabetes in Australia [46]. This reflects a lack of diabetes intervention research [47] and care [48] among Indigenous peoples more generally. Evidence of effective strategies to prevent gestational diabetes among Indigenous women, and to improve care during and after pregnancy for Indigenous women with gestational diabetes, is urgently needed. This includes collaborative mixed methods research translation strategies to develop strategies to improve postpartum care for Indigenous women with gestational diabetes and reduce the risks of implementation with limited evidence. Strategies should also be contextually and culturally relevant and, where possible, address broader socio-ecological factors [49]. The research must be underpinned by Indigenous research principles, driven by identified needs and include active community engagement.

\section{Conclusions}

Evidence of effective strategies to improve postpartum screening and to reduce the risk of developing type 2 diabetes postpartum, including strength-based approaches 
for supporting exclusive breastfeeding and healthy lifestyles, are urgently needed. This is particularly important for Indigenous women who have low rates of postpartum screening and more than a fourfold risk of developing type 2 diabetes, compared with non-Indigenous women.

\section{Acknowledgements}

Catherine Chamberlain is supported by the National Health and Medical Research Council Early Career Fellowship (1088813). The Cairns Diabetes Centre provided financial assistance to enable reviews of some medical records for this project. This project would not be possible without the support of all other members of the Project Advisory Group, including Prof. Bronwyn Fredericks, Assoc. Prof. Ashim Sinha, Dr Jacqueline Mein, Ms Bronwyn Davis and Ms Cathryn Dowey. We gratefully acknowledge support from Ms Nancy Goncalves, Ms Ann Carroll and Ms Susan Caddaye for assisting with data collection. We especially thank Ms Philippa Loane from the Clinical Informatics and Data Management Unit, Monash University, for assistance in establishing a Microsoft Access database. We appreciate and acknowledge the following organizations providing data for this project: the Health Statistics Branch (Queensland Health), Cairns Hospital Health Information Services, Sullivan and Nicolaides Laboratories and QML Pathology.

\section{Author contributions}

C. C. proposed the design, developed the methods, prepared and submitted ethics applications, developed data collection tools, linked data and prepared the draft manuscript. B. O. and S.E. contributed to study design. R.W. provided statistical advice and assistance. All authors contributed to and approved the final manuscript.

\section{Conflicts of interest}

\section{None declared.}

\section{References}

1. World Health Organization. Diagnostic Criteria and Classification of Hyperglycaemia First Detected in Pregnancy. WHO: Geneva, 2013. Available at: http://apps.who.int/iris/bitstream/ 10665/85975/1/WHO_NMH_MND_ 13.2_eng.pdf?ua $=1$ Accessed 20/4/2015

2. Ferrara A. Increasing prevalence of gestational diabetes mellitus: a public health perspective. Diabetes Care 2007; 30(S2): S141-S146.

3. HAPO Study Cooperative Research Group. Hyperglycaemia and adverse pregnancy outcomes. $N$ Engl $J$ Med 2008; 358(19): 1991-2002.

4. Bellamy L, Casas J-P, Hingorani AD, Williams D. Type 2 diabetes mellitus after gestational diabetes: a systematic review and meta-analysis. Lancet 2009; 373(9677): 1773-1779.

5. Farrell T, Neale L, Cundy T. Congenital anomalies in the offspring of women with type 1 , type 2 and gestational diabetes. Diabet Med 2002; 19(4): 322-326.

6. Naqshbandi M, Harris S, Esler J, AntwiNsiah F. Global complication rates of type 2 diabetes in Indigenous peoples: a comprehensive review. Diabetes Res Clin Pract 2008; 82(1): 1-17.

7. Kim C. Gestational diabetes and the incidence of type 2 diabetes: a systematic review. Diabetes Care 2002; 25(10): 1862-1868.

8. Mohamed N, Dooley J. Gestational diabetes and subsequent development of NIDDM in aboriginal women of northwestern Ontario. Int $J$ Circumpolar Health 1998; 57(S1): 355-358.

9. McGrath NM, Baldwin A. Further postpartum follow-up of women with gestational diabetes mellitus from Northland, New Zealand. Diabet Med 2012; 29(3): 415.

10. Benjamin E, Winters D, Mayfield J, Gohdes D. Diabetes in pregnancy in Zuni Indian women: prevalence and subsequent development of clinical diabetes after gestational diabetes. Diabetes Care 1993; 16(9): 1231-1235.

11. Steinhart JR, Sugarman JR, Connell FA. Gestational diabetes is a herald of NIDDM in Navajo women. High rate of abnormal glucose tolerance after GDM. Diabetes Care 1997; 20(6): 943-947.

12. Davis B, McLean A, Sinha AK, Falhammar H. A threefold increase in gestational diabetes over two years: review of screening practices and pregnancy outcomes in Indigenous women of Cape York, Australia. Aust N Z J Obstet Gynaecol 2013; 53(4): 363-368.

13. Queensland Health (ed). Cairns Base Hospital and associated services clinical services plan. Cairns, 2008.

14. Chamberlain C, Fredericks B, Davis B, et al. Postpartum care for Aboriginal and non-Aboriginal women with gestational diabetes mellitus across urban, rural and remote locations: a protocol for a cohort linkage study. SpringerPlus 2013; 2. doi:10.1186/2193-1801-1182-1576.

15. Hoffman L, Nolan C, Wilson DA, Oats J, Simmons D. Gestational diabetes mellitus - management guidelines. Med $J$ Aust 1998; 169(2): 93-97.

16. Depczynski B, Wong VW, Russell HD, Opi N. The impact of potential new diagnostic criteria on the prevalence of gestational diabetes mellitus in Australia. Med J Aust 2011; 195(5): 268.

17. Queensland Health and Royal Flying Doctors Service (Queensland section) (ed). Primary Clinical Care Manual (4th Edition). Queensland Health and Royal Flying Doctors Service: Cairns, 2005.

18. Queensland Health and Royal Flying Doctors Service (Queensland section) (ed). Primary Clinical Care Manual (5th Edition). Queensland Health and Royal Flying Doctors Service: Cairns, 2007.

19. Queensland Health and Royal Flying Doctors Service (Queensland Section) (ed). Primary Clinical Care Manual. 6th Edition. Cairns: Queensland Health and Royal Flying Doctors Service, 2009.

20. Colagiuri S, Davies D, Girgis S, Colagiuri R. National Evidence Based Guideline for Case Detection and Diagnosis of Type 2 Diabetes. Diabetes Australia and the NHMRC: Canberra, 2009.

21. StataCorp. Stata Statistical Software: Release 13. StataCorp LP: College Station, TX, 2013.

22. Chamberlain C, McLean A, Oats J, et al. Low rates of postpartum glucose screening among Indigenous and nonIndigenous women in Australia with gestational diabetes. Matern Child Health $J$ 2015; 19(3): 651-663. 
23. Chamberlain C, Fredericks B, McLean A, Oldenburg B, Mein J, Wolfe R. Associations with low rates of postpartum glucose screening after gestational diabetes among Indigenous and nonIndigenous Australian women. Aust N Z $J$ Public Health 2014; 39(1): 69-76.

24. Much D, Beyerlein A, Roßbauer M, Hummel S, Ziegler A-G. Beneficial effects of breastfeeding in women with gestational diabetes mellitus. $\mathrm{Mo}$ Metabol 2014; 3(3): 284-292.

25. Population and public health division: improved reporting of Aboriginal and Torres Strait Islander peoples on population datasets in New South Wales using record linkage - a feasibility study Sydney. NSW Ministry of Health, 2012.

26. Paez KA, Eggleston EM, Griffey SJ, et al. Understanding why some women with a history of gestational diabetes do not get tested for diabetes. Womens Health Issues 2014; 24(4): e373-e379.

27. Ko JY. Strategies associated with higher postpartum glucose tolerance screening rates for gestational diabetes mellitus patients. $J$ Womens Health (Larchmt) 2013; 22(8): 681-686

28. Mendez-Figueroa H, Daley J, Breault $P$, et al. Impact of an intensive follow-up program on the postpartum glucose tolerance testing rate. Arch Gynecol Obstet 2014; 289(6): 1177-1183.

29. Gupta Y, Gupta A. Post-partum screening after gestational diabetes. Lancet $D i-$ abetes Endocrinol 2013; 1(2): 90-91.

30. Kim C. Managing women with gestational diabetes mellitus in the postnatal period. Diabetes Obes Metab 2010; 12 (1): 20-25.

31. Osgood ND, Dyck RF, Grassmann WK. The inter- and intragenerational impact of gestational diabetes on the epidemic of type 2 diabetes. Am J Public Health 2011; 101(1): 173-179.

32. Ohlendorf JM. Stages of change in the trajectory of postpartum weight selfmanagement. J Obstet Gynecol Neonatal Nurs 2012; 41(1): 57-70.
33. Lie ML, Hayes L, Lewis-Barned NJ, May C, White M, Bell R. Preventing type 2 diabetes after gestational diabetes: women's experiences and implications for diabetes prevention interventions. Diabet Med 2013; 30(8): 986-993.

34. Nicklas JM, Miller LJ, Zera CA, Davis RB, Levkoff SE, Seely EW. Factors associated with depressive symptoms in the early postpartum period among women with recent gestational diabetes mellitus. Matern Child Health $J$ 2013; 17(9): 1665-1672.

35. Heslehurst N, Russell S, McCormack S, Sedgewick G, Bell R, Rankin J. Midwives perspectives of their training and education requirements in maternal obesity: a qualitative study. Midwifery 2013; 29(7): 736-744.

36. Morrison MK, Lowe JM, Collins CE. Australian women's experiences of living with gestational diabetes. Women Birth 2014; 27(1): 52-57.

37. Peacock AS, Bogossian FE, Wilkinson SA, Gibbons KS, Kim C, McIntyre HD. A randomised controlled trial to delay or prevent type 2 diabetes after gestational diabetes: walking for exercise and nutrition to prevent diabetes for you. Int $J$ Endocrinol 2015. doi:10.1155/2015/ 423717.

38. Parsons J, Ismail K, Amiel S, Forbes A. Perceptions among women with gestational diabetes. Qual Health Res 2014; 24(4): 575-85.

39. Nicklas JM, Zera CA, Seely EW, AbdulRahim ZS, Rudloff ND, Levkoff SE. Identifying postpartum intervention approaches to prevent type 2 diabetes in women with a history of gestational diabetes. BMC Pregnancy Childbirth 2011; 11: 23.

40. Dasgupta K, Da Costa D, Pillay S, et al. Strategies to optimize participation in diabetes prevention programs following gestational diabetes: a focus group study. PLoS One 2013; 8(7): e67878.

41. Reinhardt JA, van der Ploeg HP, Grzegrzulka R, Timperley JG.
Implementing lifestyle change through phone-based motivational interviewing in rural-based women with previous gestational diabetes mellitus. Health Promot J Austr 2012; 23(1): 5-9.

42. Jones EJ, Appel SJ, Eaves YD, Moneyham L, Oster RA, Ovalle F. Cardiometabolic risk, knowledge, risk perception, and self-efficacy among American Indian women with previous gestational diabetes. $J$ Obstet Gynecol Neonatal Nurs 2012; 41(2): 246-257.

43. Rimal RN. Perceived risk and selfefficacy as motivators: understanding individuals' long-term use of health information. $J$ Commun 2001; 51(4): 633-654.

44. Australian Government. National Aboriginal and Torres Strait Islander Health Plan 2013-2023. Canberra: Department of Health and Ageing, Commonwealth of Australia, 2013.

45. Tieu J. Interconception care for women with a history of gestational diabetes for improving maternal and infant outcomes. Cochrane Database Syst Rev 2013; 6: CD010211.

46. Chamberlain C, McNamara B, Williams $\mathrm{ED}$, et al. Diabetes in pregnancy among indigenous women in Australia, Canada, New Zealand and the United States: a systematic review of the evidence for screening in early pregnancy. Diabetes Metab Res Rev 2013; 29(4): 241-256.

47. McNamara BJ, Sanson-Fisher R, D’Este $\mathrm{C}$, Eades $\mathrm{S}$. Type 2 diabetes in Indigenous populations: quality of intervention research over 20 years. Prev Med 2011; 52(1): 3-9.

48. Kenyon G. Diabetes care is still failing Aboriginal Australian people. Lancet Diabetes Endocrinology 2013; 1(1): 15.

49. Milstein B, Homer J, Briss P, Burton D, Pechacek T. Why behavioral and environmental interventions are needed to improve health at lower cost Health Aff (Millwood) 2011; 30(5): 823-832.

\section{Supporting information}

Additional supporting information may be found in the online version of this article at the publisher's web site. 


\section{Author Query Form}

\section{Journal: Diabetes/Metabolism Research and Reviews}

\section{Article: dmrr_2715}

Dear Author,

During the copyediting of your paper, the following queries arose. Please respond to these by annotating your proofs with the necessary changes/additions.

- If you intend to annotate your proof electronically, please refer to the E-annotation guidelines.

- If you intend to annotate your proof by means of hard-copy mark-up, please use the standard proofing marks. If manually writing corrections on your proof and returning it by fax, do not write too close to the edge of the paper. Please remember that illegible mark-ups may delay publication.

Whether you opt for hard-copy or electronic annotation of your proofs, we recommend that you provide additional clarification of answers to queries by entering your answers on the query sheet, in addition to the text mark-up.

\begin{tabular}{|c|l|c|}
\hline Query No. & \multicolumn{1}{|c|}{ Query } & Remark \\
\hline Q1 & $\begin{array}{l}\text { AUTHOR: Please confirm that given names (red) and surnames/family names } \\
\text { (green) have been identified correctly. }\end{array}$ & \\
\hline Q2 & AUTHOR: Please check that authors and their affiliations are correct. & \\
\hline Q3 & AUTHOR: Please provide the name of publisher for Reference 13. & \\
\hline Q4 & AUTHOR: Please check if Reference 44 is captured correctly. & \\
\hline
\end{tabular}


Required software to e-Annotate PDFs: Adobe Acrobat Professional or Adobe Reader (version 7.0 or above). (Note that this document uses screenshots from Adobe Reader $\mathrm{X}$ )

The latest version of Acrobat Reader can be downloaded for free at: http://get.adobe.com/uk/reader/

Once you have Acrobat Reader open on your computer, click on the Comment tab at the right of the toolbar:

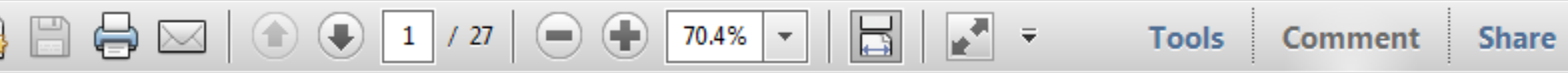

This will open up a panel down the right side of the document. The majority of tools you will use for annotating your proof will be in the Annotations section, pictured opposite. We've picked out some of these tools below:

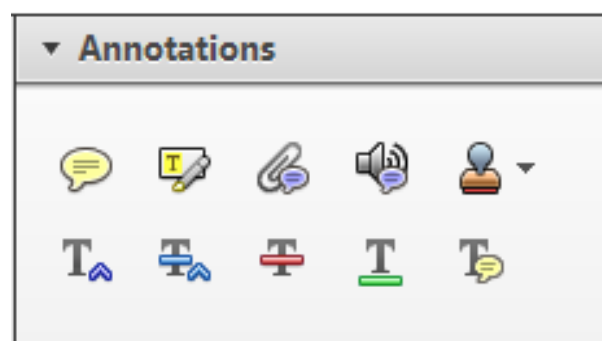

1. Replace (Ins) Tool - for replacing text.

Strikes a line through text and opens up a text box where replacement text can be entered.

How to use it

- Highlight a word or sentence.

- Click on the Replace (Ins) icon in the Annotations section.

- Type the replacement text into the blue box that appears.

Idard tramework for the analysis of $\mathrm{m}$ icy-Nevertheless, it also led to exog،

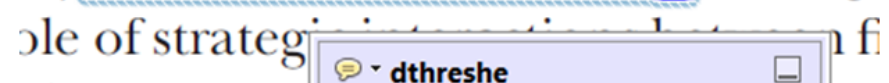
aber of comp 08/06/2011 15:58:17 is that the s1 nain compo: be level, are exc nc

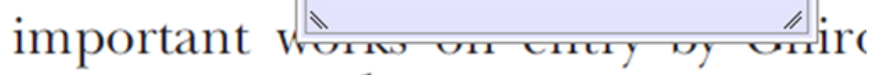
M heneferth) ${ }^{1}$ we anen the "hlarl $\mathrm{l}$

3. Add note to text Tool - for highlighting a section to be changed to bold or italic.

T Highlights text in yellow and opens up a text box where comments can be entered.

\section{How to use it}

- Highlight the relevant section of text.

- Click on the Add note to text icon in the Annotations section.

- Type instruction on what should be changed regarding the text into the yellow box that appears.

namic responses of mark ups ent with the VAR evidence

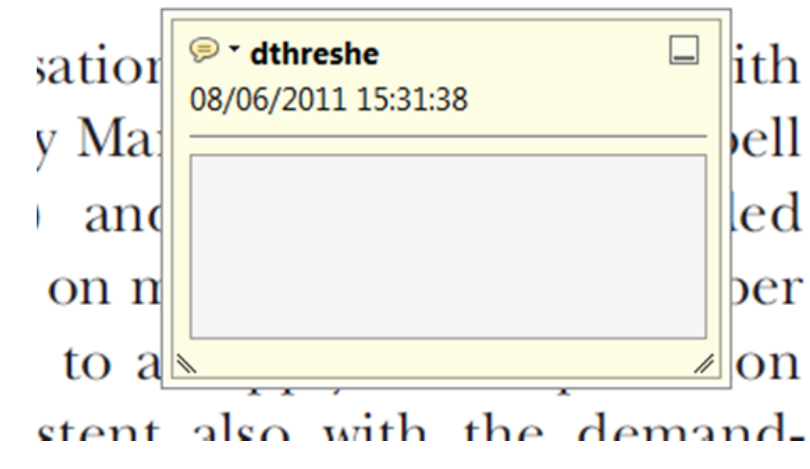

2. Strikethrough (Del) Tool - for deleting text.

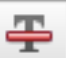

Strikes a red line through text that is to be deleted.

How to use it

- Highlight a word or sentence.

- Click on the Strikethrough (Del) icon in the Annotations section.

there is no room tor extra prohts al c ups are zero and the number of ret) values are not determined by Blanchard and Kiyotaki (1987), sfect competition in general equilil ts of aggregate demand and supply lassical framework assuming monol eph on evorenous number of firme

4. Add sticky note Tool - for making notes at specific points in the text.

Marks a point in the proof where a comment needs to be highlighted.

How to use it

- Click on the Add sticky note icon in the Annotations section.

- Click at the point in the proof where the comment should be inserted.

- Type the comment into the yellow box that appears.

iaisu airu suppiy sirucks. hivsl ui

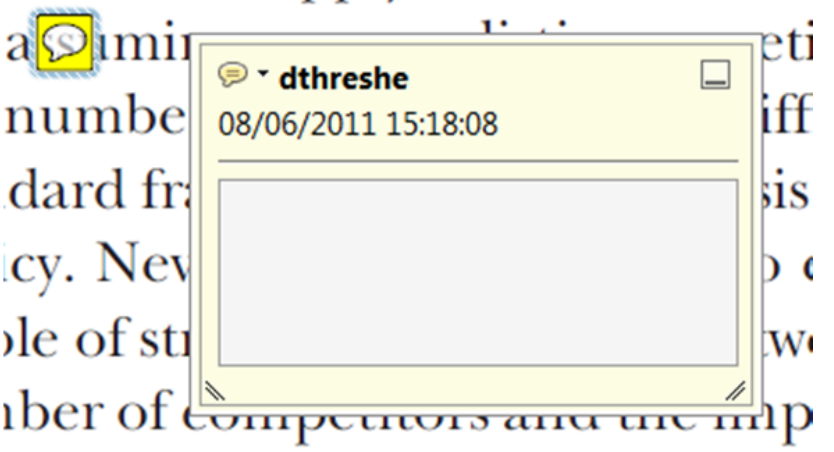

is that the structure of the secto. 
5. Attach File Tool - for inserting large amounts of text or replacement figures.

Inserts an icon linking to the attached file in the appropriate pace in the text.

How to use it

- Click on the Attach File icon in the Annotations section.

- Click on the proof to where you'd like the attached file to be linked.

- Select the file to be attached from your computer or network.

- Select the colour and type of icon that will appear in the proof. Click OK.

E N D

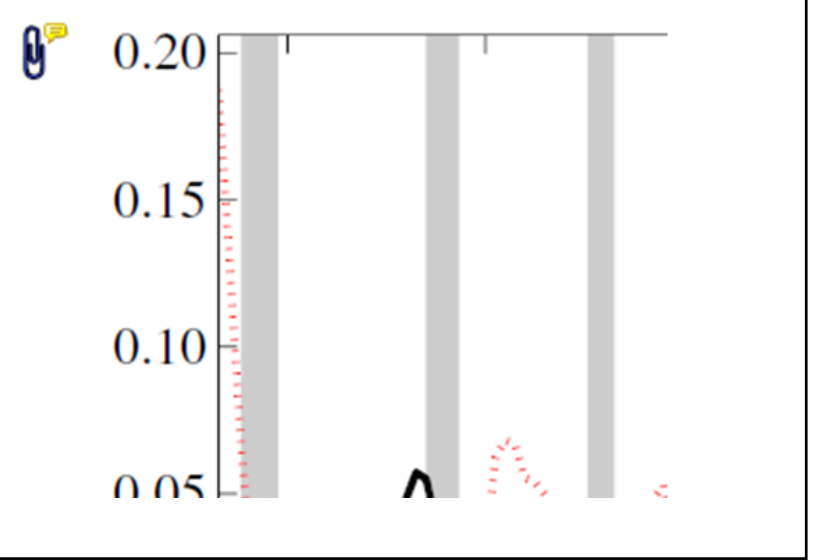

6. Add stamp Tool - for approving a proof if no corrections are required.

- Inserts a selected stamp onto an appropriate place in the proof.

\section{How to use it}

- Click on the Add stamp icon in the Annotations section.

- $\quad$ Select the stamp you want to use. (The Approved stamp is usually available directly in the menu that appears).

- Click on the proof where you'd like the stamp to appear. (Where a proof is to be approved as it is, this would normally be on the first page).

or the business cycie, starting with the on perfect competition, constant ret

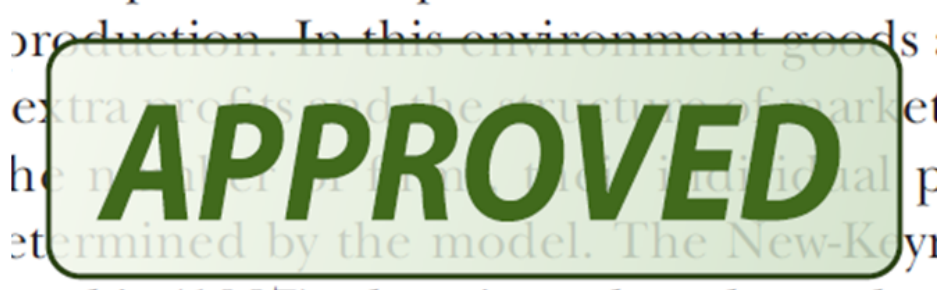
otaki (1987), has introduced produc general equilibrium models with nomin:

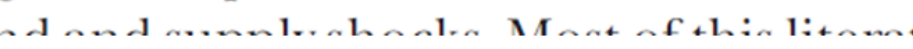

- Drawing Markups

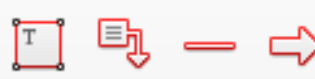

$0 \bigcirc \sqrt{6} \otimes$

\section{How to use it}

- Click on one of the shapes in the Drawing Markups section.

- Click on the proof at the relevant point and draw the selected shape with the cursor.

- To add a comment to the drawn shape, move the cursor over the shape until an arrowhead appears.

- Double click on the shape and type any text in the red box that appears.
7. Drawing Markups Tools - for drawing shapes, lines and freeform annotations on proofs and commenting on these marks.

Allows shapes, lines and freeform annotations to be drawn on proofs and for comment to be made on these marks.

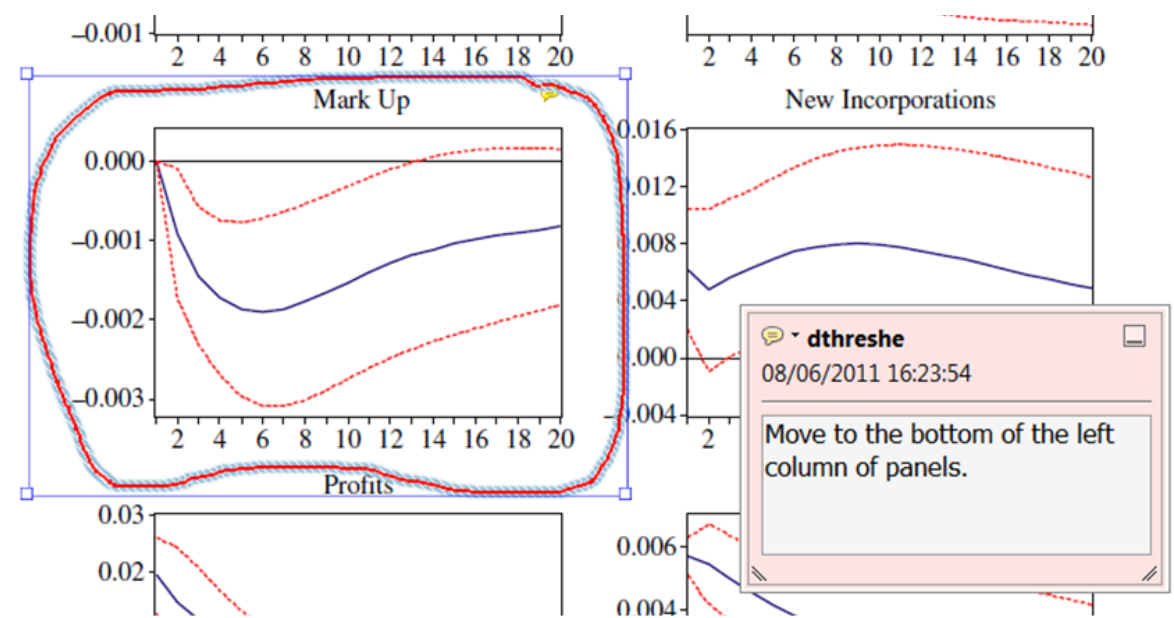

For further information on how to annotate proofs, click on the Help menu to reveal a list of further options:

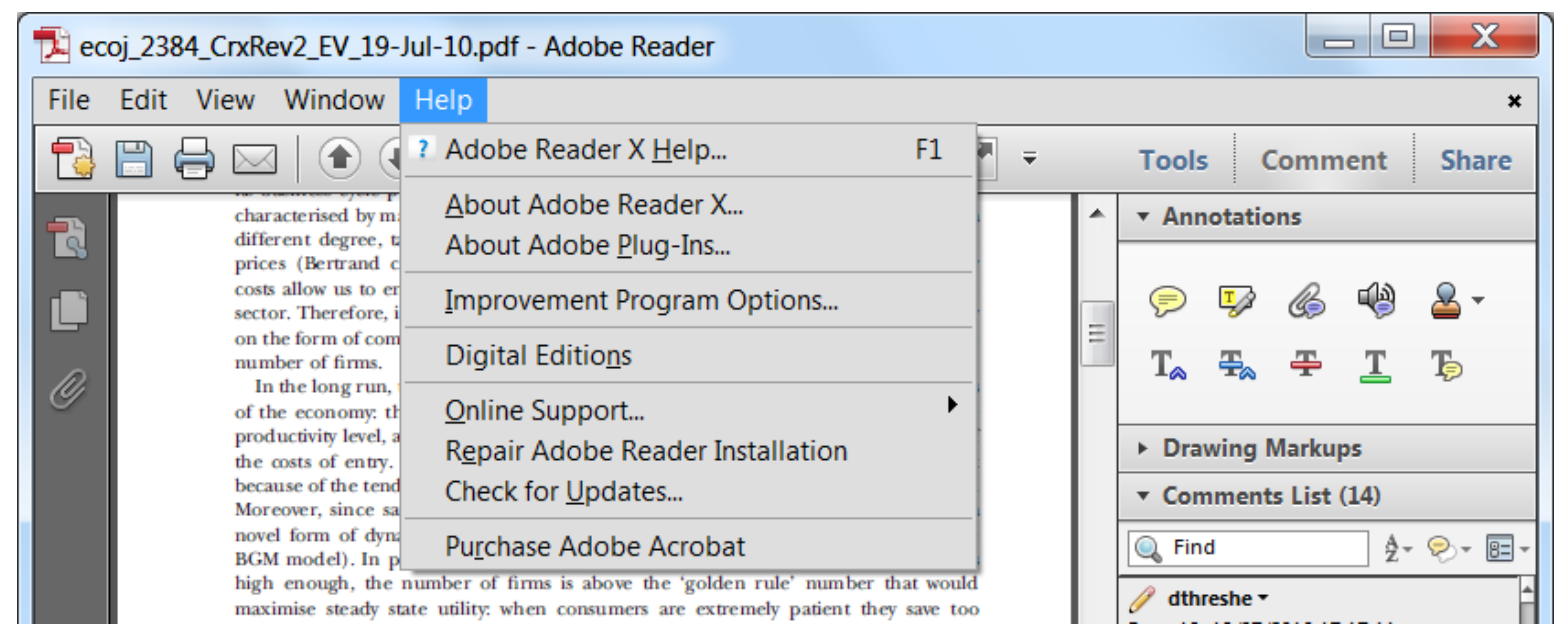

